\title{
Zu dieser Studie
}

Das ISR legt eine weitere Studie zum Themenbereich räumliche Innovationsforschung vor und damit zur Rolle von Universitäten und den von ihnen ausgehenden wirtschaftlichen Aktivitäten in einer wissensbasierten Gesellschaft. Nach der Wertschöpfungsstudie der Universität Wien bzw. der Wiener Hochschulen befasst sich die vorliegende Analyse mit dem F\&E-Sektor in Wien, mit seinen Strukturen, seinen inhaltlichen Ausrichtungen sowie besonders mit seinem Standortmuster. Rund 1.500 in Wien angesiedelte öffentliche und private Forschungseinrichtungen sowie Unternehmen mit betrieblicher Forschung stellten die Grundlage der räumlichen Verteilungsanalyse dar. Zusätzlich fanden 14 ausführliche strukturierte Interviews mit Vertretern der privaten und öffentlichen Forschungseinrichtungen des Life-Science- und IKT-Clusters sowie mit weiteren Experten des F\&E-Sektors zu den Themenbereichen Förderungen, Standorte und institutionelle Verankerungen statt.

Die Studie kann mit zahlreichen interessanten empirischen Resultaten aufwarten. Sie dokumentiert die Bedeutung Wiens für den F\&E-Sektor in Österreich. Sie zeigt des Weiteren auf, in welchen europäischen Metropolen der Stellenwert des F\&E-Sektors über jenem der Stadt Wien liegt und sie identifiziert die räumlichen Standortmuster des F\&E-Sektors im Allgemeinen und der Life Sciences sowie der IKT im Speziellen. In diesem Zusammenhang ist vor allem eines hervorzuheben: Die Befragung hat deutlich gemacht, dass der ,richtige“ Standort für die Zufriedenheit der Forscher und Forscherinnen und für den wirtschaftlichen Erfolg des Unternehmens entscheidend ist. Räumliche Nähe und eine gute Erreichbarkeit senken die Transaktionskosten bei der Weitergabe von Informationen und schaffen ein innovationsförderndes Arbeitsumfeld. Die Stadtentwicklungspolitik sollte daher mit einer F\&E-Politik stärker als bisher Hand in Hand gehen und beide sollten einander gegenseitig beeinflussen. Ein übergeordneter Masterplan zur Standortentwicklung des F\&E Sektors, in Abstimmung mit der FITStrategie der Stadt Wien und dem Stadtentwicklungsplan, wird in diesem Zusammenhang besonders empfohlen.

Die Initiative zu diesem Forschungsprojekt ging abermals vom Beauftragten der Stadt Wien für Universitäten und Forschung, Prof. Alexander VAN DER BELLEN, aus, dem dafür auch zu danken ist. Solche Initiativen sind wichtig, um das Thema weiterzuentwickeln und die Politik damit zu Denkanstößen zum künftigen Handeln aufzufordern. Robert MusiL und Jakob EDER haben diese Studie theoretisch und empirisch begleitet und inhaltlich durchgeführt. Ihnen gebührt ebenso Dank wie Statistik Austria für die Durchführung einer Sonderauswertung sowie den zahlreichen Gesprächspartnern für ihre wertvollen Inputs.

Univ.-Prof. Dr. Heinz FassmanN

Direktor des Instituts für Stadt- und Regionalforschung 
